\title{
Management of a solitary pulmonary nodule
}

\section{Philippe Grenier MD}

The solitary pulmonary nodule (SPN) is a common radiologic abnormality. An estimated 150000 SPNs are detected annually in the US and are often discovered incidentally at chest radiography or CT. These nodules are caused by a variety of disorders, including neoplasms, infection, granulomas, intrapulmonary lymph node, and vascular and congenital abnormalities. Although most SPNs have benign cause, 35 - $40 \%$ of these nodules are malignant, many of them representing stage I lung cancer that must be distinguished from benign nodules in an expeditious and cost effective manner. Small pulmonary nodules (less than one centimeter) of indeterminate etiology are being detected with increasing frequency owing to greater use of computed tomography. Since the advance of helical CT scanning, even more pulmonary nodules are being detected.

When a SPN is depicted, helical thin collimation CT scans through the nodule should be obtained prior to contrast infusion. The use of thin slice thickness is essential for assessing morphology and attenuation. Then obtaining a chest study including the upper abdomen is also important for staging purposes and for detecting other additional nodules. In case of multiple nodules present in CT examination, every individual nodule has to be analyzed independently. When the number of nodules present is superior to 6, the probability of granulomatous or metastatic disease is very high and the management is totally different and excluded from this chapter.

\section{Morphology and density at CT}

A few types of focal lesions have morphologic characteristics typical enough to allow a specific diagnosis to be made on HRCT. Examples include arteriovenous pulmonary malformations, fungus ball, rounded atelectasis and mucus plugs.

In most of the cases, the morphology of the nodule is not characteristic enough to identify the lesion, but the analysis of the size, margins, content, and density is the first step that can help differentiate benign and malignant lesions.

\section{Size and margins}

Generally, the smaller the nodule, the more likely it is to be benign. Nodules larger than $1 \mathrm{~cm}$ are more likely to be malignant, whereas nodules smaller than $0.5 \mathrm{~cm}$ are more likely to be benign. However, small size alone does not exclude lung cancer because $15 \%$ of malignant nodules are less than $1 \mathrm{~cm}$.

Margins and contours have been classified as smooth, lobulated, irregular or spiculated. Although most nodules with smooth well-defined margins are benign, these features are not diagnostic for a benign cause: $21 \%$ of malignant nodules have well-defined margins. Metastatic carcinomas are often smooth in contour. A lobulated contour implies an even growth which is associated with malignancy. Lobulation however also occurs in up to $25 \%$ of benign nodules. A nodule with an irregular or spiculated margin is likely to be malignant although occasionally it can be due to focal organizing pneumonia or plasma cell granuloma.

\section{Content}

Air bronchogram or focal bubblelike lucencies, (pseudocavitation), within a nodule are more common in malignancy $(30 \%)$ than in benign nodules $(5 \%)$ and are more suggestive of adenocarcinoma, or bronchioloalveolar cell carcinoma.

Cavitation is also more common in malignant nodules than benign, but focal benign pulmonary lesions, such as lung abscess can cavitate as well. Benign cavitary lesions generally have smooth, thin walls whereas malignant nodules typically have thick, irregular walls. Most nodules with a wall thickness greater than 15 $\mathrm{mm}$ are malignant, whereas those with a wall thickness less than $4 \mathrm{~mm}$ are usually benign. However, there is significant overlap and wall thickness alone cannot be used to confidently differentiate benign from malignant cavitary nodules.

Calcification: The detection of calcification and assessment of its pattern is an important step to differentiate benign and malignant nodules. There are four benign patterns of calcifications: diffuse, solid and central, laminated, and popcorn like. The first three are typically seen with prior infections, particularly histoplasmosis or tuberculosis. Popcorn like calcification is characteristic of chondroid cal- 
cification in a hamartoma. When present, these patterns of calcification are reliable indicators of a benign cause, although these lesions should be followed radiographically in most cases, unless the calcification is diffuse and dense. Eccentric or stippled calcifications are highly suggestive of malignancy.

Densely calcified SPNs in patient with a history of primary bone-forming malignancy should not be automatically interpreted as benign lesions. A small central calcification is insufficient for determining the nodule to be benign if the nodule is larger than $2.5 \mathrm{~cm}$ with spiculated margins because of the likelihood of cancer in patients with such nodules.

\section{Density}

Most nodules are of soft tissue density (solid nodules). Some others are hazy dense, less dense than vessels, taking a ground glass appearance (non solid nodules). When they are less than $5 \mathrm{~mm}$ in diameter, these ground glass areas are most likely benign (atypical adenomatous hyperplasia). Beyond $5 \mathrm{~mm}$, they can be malignant (non mucinous bronchioloalveolar cell carcinoma or adenocarcinoma), and they must be regarded with high suspicion of malignancy when larger than $10 \mathrm{~mm}$. Presence of soft tissue component within the ground glass opacity (mixed nodule) is also a feature of high suspicion of malignancy (adenocarcinoma).

\section{Classification of nodules and diagnostic strategies}

According to its appearance (mor-

Table 1. CT criteria of benign nodules

- Diffuse and intense calcification

- Converging vessels (pulmonary arteriovenous malformation, rounded atelectatis)

- Fat collection ( \pm pop corn calcification)

- Benign pattern of calcification (central or laminated)

Table 2. CT criteria of high suspicion of malignancy

- Size $>20 \mathrm{~mm}$

- Spiculated contours

- Air bronchogram or bubble like lucencies

- Malignant pattern of calcification (eccentric, stippled)

- Non solid nodule (focal GGO) $>10 \mathrm{~mm}$

- Mixed nodule (GGO+soft tissue)

\section{Table 3. CT criteria of indeterminate nodule}

- Non solid (focal GGO) $<10 \mathrm{~mm}$

- Solid $<20 \mathrm{~mm}$

- Non spiculated contours

- Without air bronchogram of bubble like lucencies

- Without malignant calcification

- Without fat and benign calcification

phology and density) on CT scans, the lung nodule can be classified as benign, highly suspected of malignancy or indeterminate. Nodules considered as benign are those with diffuse calcification or those having a criterion of specific benign disease (Table 1). No additional examination (diffuse calcification) or only radiographic follow up until 2 years stability is requested.

Nodules showing any of the criteria defining high suspicion of malignancy (Table 2) must be biopsied (commonly by videoassisted-thoracoscopic surgery [VATS]).

The indeterminate nodules $(70 \%)$ are those presenting with the CT criteria reported in Table 3 . The differentiation between malignant and benign nodules can be done by different diagnostic approaches including growth rate assessment, contrast enhanced CT, FDG-PET, biopsies, or VATS.

\section{Growth rate assessment}

Doubling time for most malignant nodules is between 30 and 400 days and results in $26 \%$ increase in nodule diameter. Nodules that double either more rapidly or more slowly typically have a benign cause. Stability at chest radiograph or CT over a 2-year period implies a doubling time of at least 730 days and is generally considered to be a reliable indicator of a benign cause. It can be difficult to reliably detect growth in small $(10 \mathrm{~mm})$ nodules. For example, a $5 \mathrm{~mm}$ nodule can double in volume but its diameter will increase by only $1.25 \mathrm{~mm}$. This 1.25 $\mathrm{mm}$ change in diameter cannot be reliably detected with CT. To overcome this limitation, it has been proposed that the growth rate of small nodules be assessed with serial measurements of volume rather than diameter. Specific softwares of image analysis allowing the automatic seg- 
mentation of the contour on CT scans, 3D reconstructions of the nodules, and calculation of nodule volume, have been prepared by industry and are under evaluation.

\section{Contrast enhanced CT}

The degree of enhancement has proven to be directly related to the likelihood of malignancy and the vascularity of the nodule. The technique (only for nodules larger than $7 \mathrm{~mm}$ ) consists of thin section helical CT performed through the nodule before and after (every $30 \mathrm{sec}$ for $5 \mathrm{~min}$ ), intravenous iodinated contrast material $(300 \mathrm{mg} / \mathrm{ml}$ at $2 \mathrm{ml} / \mathrm{sec}$, total dose $420 \mathrm{mg} / \mathrm{Kg}$ ). Nodular enhancement absent or less than $15 \mathrm{HU}$ after contrast material is strongly predictive of a benign lesion (sensitivity $98 \%$, specificity $73 \%$ ). The low specificity is due to enhancement observed in some hamartomas and inflammatory lesions. This technique may help select patients for biopsy or surgery (if enhancement is present) or follow-up (if enhancement is absent).

\section{FDG-PET}

Increased glucose metabolism in tumors results in increased uptake, trapping and accumulation of FDG, permitting differentiation of benign and malignant nodules. The sensitivity, specificity and accuracy of FDGPET in the diagnosis of benign nodules are $96 \%, 88 \%$ and $94 \%$ respectively. Lesions with low FDG uptake may be considered benign. The high specificity of FDG-PET for the diagnosis of benign lesions has important clinical utility. However these lesions should be followed radiologically because of false negative results due to small size (lower than $10 \mathrm{~mm}$ ) and some malignant lesions such as carcinoid and bronchoalveolar cell carcinoma. SPN with increased FDG uptake should be considered malignant, although false positive results can be obtained with infections or inflammatory processes such as active tuberculosis, histoplasmosis and rheumatoid nodules.

\section{Biopsy}

Biopsy of a lung nodule may be necessary in some cases. Several options are available. These include bronchoscopy, needle aspiration lung biopsy, video-assisted thoracoscopic surgery (VATS) or thoracotomy. To some degree, imaging studies can be valuable in making this choice. If the SPN is related to a narrowed or obstructed bronchus or a bronchus is visible within the nodule (a positive bronchus sign) or if an endobronchial lesion is detected with CT, bronchoscopy directed to the proper level is appropriate and often will be diagnostic. In such cases, $\mathrm{CT}$ can guide the biopsy attempt. If a positive bronchus sign is not present or the nodule is peripheral, needle aspiration biopsy is more appropriate. CT can be helpful in planning the biopsy. It can indicate the depth of the lesion and the needle can be appropriately marked. In addition, the biopsy approach may be planned to avoid that the path of the needle crosses a bullae or a fissure. The risk of pneumothorax in such cases is increased and a different approach may be used. Lesions smaller than 1 $\mathrm{cm}$ can be biopsied percutaneously, although the minimum size varies with the skill of the radiologist. Needle aspiration yields malignant cells in more than $90 \%$ of neoplastic nodules. This percentage can be optimized by having a cytopathologist on site at the time of the biopsy and repeating the biopsy if the specimens are negative. Some investigators, because of the lack of availability of a cytopathologist, prefer to use cutting coaxial nee- dles that provide larger samples permitting histological studies. These techniques improve the accuracy of specific diagnosis of benign lesions without any significant increase in the number of complications. A non specific negative biopsy cannot be taken into account to indicate that no cancer is present. CT can also be used in conjunction with VATS. Localization of the tumor can be done preoperatively using dye or a hooked wire.

\section{Diagnostic approach of indeterminate nodule}

The choice between the different diagnostic approaches of an indeterminate nodule depends on the clinical probably of malignancy and the nodule size.

- < $5 \mathrm{~mm}$ : Follow-up CT at 12 and 24 months consider biopsy or removal for nodules that increase in volume (more than $15 \%$ )

- 5-10 mm: Follow-up CT at 3, 6, 12, and 24 months. Consider biopsy or removal for nodules that increase in volume (more than 15\%)

- >10 mm: Consider biopsy. As an alternative, PET or contrastenhanced CT can be done.

\section{Suggested reading}

1. Aberle DR, Gamsu G, Henschke CI, Naidich DP, Swensen SJ. A consensus statement of the Society of Thoracic Radiology. Screening for lung cancer with helical computed tomography. J Thorac Imaging 2001; 16: 65-68.

2. Erasmus JJ, Connolly JE, McAdams HP, Roggli VL. Solitary pulmonary nodules: Part I. Morphologic evaluation for differentiation of benign and malignant lesions. Radiographics 2000; 20: 43-58.

3. Erasmus JJ, McAdams HP, Collolly JE. Solitary pulmonary nodules: Part II. Evaluation of the indeterminate nodule. Radiographics 2000; 20: 59-66

4. Ginsberg MS, Griff SK, Go BD, Yoo $\mathrm{HH}$, Schwartz LH, Panicek DM. Pulmonary nodules resected at video-asisted thoracoscopic surgery: etiology in 426 patients. Radiology 1999; 213: 277-282. 